\title{
Uterus Absent
}

National Cancer Institute

\section{Source}

National Cancer Institute. Uterus Absent. NCI Thesaurus. Code C138958.

An indication that an individual's uterus is not present. 